\title{
Ethische Wegweiser für Prognosestellung und Psychotherapie im Strafrecht sowie Straf- und Massnahmenvollzug
}

\author{
Die Psychiatrie hat sich zunehmend dazu hergegeben, ordnungspolitische Extrem- \\ forderungen der Politik, welche die strafrechtlichen Instanzen in Beachtung recht- \\ licher und ethischer Maximen nicht erfüllen können, dennoch in die Tat umzusetzen. \\ Die psychotherapeutische Behandlung im Strafvollzug hat sich jeglicher Repression \\ zu enthalten.
}

Mario Gmür

\section{«Die demütigende Wirkung der Strafe kann beim Täter} Trotzreaktionen deliktischer Art auslösen.»

Korrespondenz:

PD Dr. med. Mario Gmür Rämistrasse 3

CH-8001 Zürich

mario_gmuer[at]bluewin.ch
Das Strafrecht soll neben der ausgleichenden Funktion und einer generalpräventiven Wirkung einen individualpräventiven Erfolg zeitigen. Dieser ist quasi von therapeutischer Art: Indem der Täter durch die Sanktion und Strafverbüssung beeindruckt wird, soll er von Rückfällen abgehalten werden. Dies ist bei vielen Verurteilten auch der Fall. Es gibt allerdings Gründe, weshalb diese Erwartung oft enttäuscht wird. Die demütigende Wirkung der Strafe kann beim Täter Trotzreaktionen deliktischer Art auslösen und die schlechte Gesellschaft von Mittätern ihn auf den Geschmack zu neuen kriminellen Handlungen bringen. Ausserdem können psychische Störungen die Ursache deliktischen Verhaltens sein, denen mit Strafe nicht beizukommen ist. Hier ist die Psychiatrie gefragt, um die Indikation zur Behandlung zu prüfen und diese gegebenenfalls durchzuführen, als ambulante oder stationäre Massnahme. Diese tritt teilweise oder ganz an die Stelle der Strafe. zunehmend dazu hergegeben, ordnungspolitische Extremforderungen der Politik, welche die strafrechtlichen Instanzen in Beachtung rechtlicher und ethischer Maximen nicht erfüllen können, dennoch in die Tat umzusetzen. Das betrifft den Bereich der Prognosestellung und der therapeutischen Massnahmen und wirft wissenschaftliche und ethische Fragen auf.

Die Proliferation der Psychiatrie im Strafrechtsbereich kommt vor allem dadurch zustande, dass sie ihre Zuständigkeit immer mehr von der Beurteilung psychisch schwer gestörter Delinquenten auf die mehr oder weniger gewöhnlichen Täter ausweitet. Diese laufen aufgrund ihrer Neigung zu Normverstössen
Jalons éthiques pour la pose du pronostic et le traitement psychothérapeutique en droit pénal et lors de l'exécution de peines et mesures

Le pronostic et le traitement psychiatriques ne doivent pas servir à satisfaire des revendications politiques extrêmes mais doivent au contraire respecter les principes éthiques, y compris en psychiatrie forensique. Les résultats des outils pronostiques (par ex. Fotres, VRAG) ne doivent être utilisés qu'à titre général et non comme méthodes de test pour des cas particuliers. Les traitements psychothérapeutiques dispensés en prison ne doivent en aucun cas être répressifs et il convient notamment d'éviter toute menace de conséquences à caractère pénal. Le secret médical doit être garanti et la sphère privée du patient respectée. Les psychothérapies sans perspectives sont proscrites et les droits garantis par la procédure pénale doivent être respectés. Le traitement psychothérapeutique a une visée purement curative et en aucun cas punitive, mais il ne doit pas non plus occulter le potentiel préventif de la peine au plan individuel. Enfin, il s'agit de prévoir une réglementation disciplinaire et pénale en cas de non-respect des principes éthiques par le personnel soignant. 
seit ihrer Jugend in der Regel unter der Diagnose «Persönlichkeitsstörung».

\section{Überschätzte Prognoseinstrumente}

Die Aussagekraft von Prognoseinstrumenten (z.B. FOTRES, VRAG) wird überschätzt. Die Zahlen zur Rückfallhäufigkeit sind als allgemeine Hintergrundinformationen zwar relevante Orientierungshilfen. Sie werden aber regelmässig missbraucht, indem sie im psychiatrischen Gutachten als Testverfahren deklariert und auf den Einzelfall angewendet werden. Dies wäre nur erlaubt, wenn die zugrundeliegenden Verlaufsuntersuchungen nicht nur an einigen Hundert, sondern an Tausenden von Delinquenten durchgeführt worden wären, um genügend grosse Untergruppen von Untersuchten bilden zu können, welche die gleichen Merkmalen bezüglich Biographie und Persönlichkeit wie der Einzelfall aufweisen. Es liegt ein Wissenschaftsbetrug vor, wenn der Richter diese Täuschung nicht durchschauen kann und durch den Anschein von hoher Wissenschaftlichkeit dazu verführt wird, sein folgenschweres Urteil schwergewichtig auf die ihm präsentierten Prognoseergebnisse abzustützen. Die individuelle Bestimmung der Rückfallwahrscheinlichkeit in Form einer Prozentzahl (zum Beispiel 64\% in 7 Jahren) ersetzt das voluntaristische Modell, das an den Besserungswillen des Täters appelliert, durch ein deterministisches Modell, das den Rechtsbrecher an seiner Eigenverantwortung für sein Tun zweifeln lässt. Dem individualpräventiv-bessernden Potential der Strafe bricht dadurch das Fundament weg.

\section{Die Aussagekraft von Prognose- instrumenten wird überschätzt.}

Die Relevanz der errechneten Prognoseziffern wird allein schon dadurch stark relativiert, dass die Prognose der Rückfälligkeit eines Täters bereits durch das Fehlen resp. Beheben eines einzigen Faktors, der ein obligates Glied in der Kausalkette darstellt, nicht mehr gültig ist. Dies trifft etwa dann zu, wenn der Begutachtete nur unter Alkoholeinfluss oder nur bei Arbeitslosigkeit oder nur in einer spezifischen Lebensoder Beziehungssituation delinquiert.

Die Verlässlichkeit von Prognoseinstrumenten ist ausserdem wegen der ungenügenden Interrater-Übereinstimmung ausserhalb einer stabilen Forschungsgruppe, also in der freien Wildbahn der forensischpsychiatrischen Gutachtertätigkeit, gering.

\section{Repressive Psychotherapie}

Die Durchführung einer Psychotherapie hat auch bei Massnahmepatienten fachlichen und ethischen Qualitätskriterien genau so zu genügen wie bei allen anderen Patienten. Über die Erfolgsaussichten einer Behandlung sind alle, die daran interessiert sind, auch Justizbehörden und die Öffentlichkeit, zu informieren, damit keine unrealistischen Erwartungen daran geknüpft werden, die Enttäuschungsreaktionen auslösen können, die oft an Patient und Therapeut abreagiert werden. Wie der Kriminologe M. Killias immer wieder in öffentlichen Debatten moniert, gibt es kaum befriedigende Untersuchungen, welche die Überlegenheit von Therapien im Strafvollzug gegenüber Spontanverläufen überzeugend nachweisen. Während naturgemäss Behandlungen mit einfachem Zusammenhang zwischen Wirkung und Erfolg, wie z. B. die Neuroleptika-Verabreichung bei Schizophrenen, Lithium-Prophylaxe bei manisch-depressivem Kranksein oder Androkur bei abnormem Sexualtrieb bei guter Kooperation gute Erfolgsaussichten aufweisen, ist für die Behandlung von Persönlichkeitsstörungen keine Methode von durchschlagendem Erfolg bekannt. Wird zudem eine Behandlung nicht mit optimaler Methode angewendet, kann sie gar eine Verschlimmbesserung bewirken und einen schlechteren Verlauf zeigen als ein normaler Strafvollzug in guter Atmosphäre, der den Eigenheiten und der Verletzlichkeit des Verurteilten Rechnung trägt.

Unvereinbar mit fachlichen und ethischen Anforderungen ist jegliche repressive Anwendung von Psychotherapie. Allenfalls darf eine solche ausnahmsweise und für eine beschränkte Zeit in einem Ausmass und Umfang erfolgen, wie sie im Rahmen des Erwachsenenschutzrechts geduldet ist. Im Übrigen ist der Persönlichkeitsschutz zu gewährleisten. Das Recht zur Einschränkung der Privat- und Geheimsphäre steht ausschliesslich der zuständigen Behörde zu, der notabene eine Verletzung der Intimsphäre ebenfalls strikte untersagt ist.

Wo das Gefühlsleben eines Massnahmepatienten bearbeitet wird, ist das Arztgeheimnis zugunsten des Behandelten uneingeschränkt zu gewährleisten. Jedwede Aufforderung zu einer psychotherapeutischen Behandlung oder zur Öffnung des Patienten gegenüber dem Therapeuten unter Androhung von Repressalien wie Verwahrung, Verweigerung von Ausgängen, Disziplinarstrafen etc. ist sittenwidrig. Psychotherapie als «Beichte ohne Berufsgeheimnis» ist unstatthaft. Um möglichst viele Straftäter zu erreichen, sind eine therapeutische Methodenvielfalt und eine freie Arztwahl in einem Umfang wie es dem Strafund Massnahmenvollzug organisatorisch zumutbar ist, anzubieten.

Inakzeptabel ist ferner eine Psychotherapie ohne Perspektive, das heisst ohne Aussicht auf adäquate Erfolge. Beim Massnahmepatienten, wo die Verbesserung der Legalprognose Zweck der Behandlung ist, schliesst dies auch die Einlösung des Versprechens auf Hafterleichterung resp. Entlassung bei gutem Verlauf ein. Besonders verwerflich ist es, wenn eine Psychotherapie pro forma als taktisches Manöver zur Belegung ihres Misserfolges und damit zur Rechtfertigung 


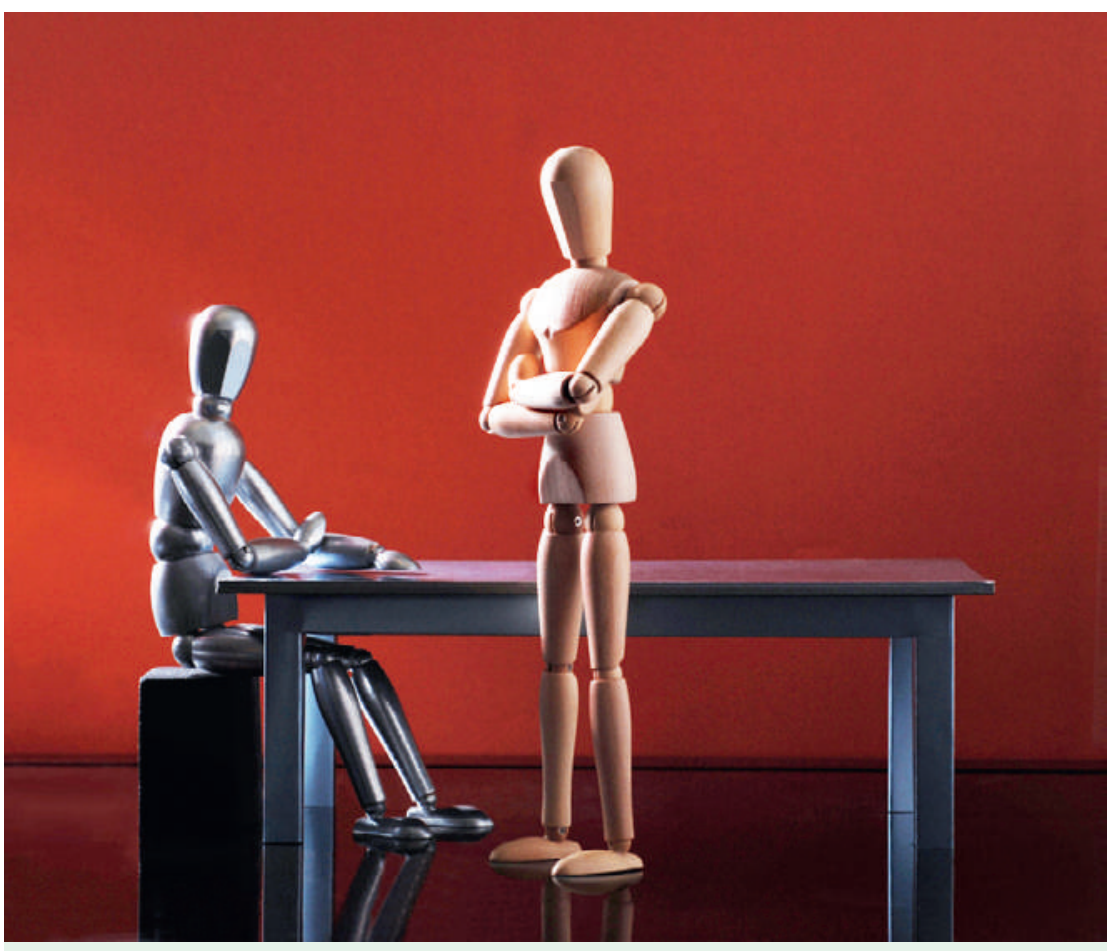

Die Aufforderung zu einer psychotherapeutischen Behandlung unter Androhung von Repressalien ist sittenwidrig. werden. Die drangsalierenden und persönlichkeitsverletzenden Interventionen und die massive Verlängerung der Freiheitseinschränkung aufgrund einer psychiatrischen Indikation werden als Strafverschärfung erlebt. Der Täter erhält den Eindruck, er werde nicht für die von ihm begangene Tat bestraft, sondern für die aus seinen biografischen Daten errechnete Gefährlichkeit. Die Brüskierung seines Gerechtigkeitsgefühls erschüttert seine Akzeptanz der Strafe und bringt die individualpräventive Funktion der Strafe bzw. Massnahme um ihre Chance.

Wie absurd diese Konstellation sein kann, zeigt sich bei einem nicht geständigen Täter, der sich in der Lage sieht, ein falsches Geständnis abzulegen und eine Kooperation in der Psychotherapie vorzuspielen, um sich den Weg in die Freiheit durch Täuschung des Psychotherapeuten zu bahnen. Die Psychotherapie bietet sich als Erfüllungsgehilfe einer totalitären Rechtsauffassung an, wenn mit ihrer Hilfe Verfahrensrechte ausser Kraft gesetzt werden, die in der Strafuntersuchung garantiert sind.

Wichtigste Forderungen an die forensische Psychiatrie sind:

- Forschungsergebnisse von sogenannten Prognoseinstrumenten dürfen nur als Hintergrundinformationen und nicht als Testverfahren für den Einzelfall verwendet werden.

- Die psychotherapeutische Behandlung im Strafvollzug hat sich jeglicher Repression zu enthalten. Insbesondere sind Androhung von Konsequenzen mit Strafcharakter zu vermeiden, das Arztgeheimnis gegenüber dem Patienten einzuhalten und dessen Intimsphäre zu respektieren. Im Strafverfahren garantierte Rechte dürfen nicht unterlaufen werden.

- Die psychotherapeutische Massnahme hat ausschliesslich einen kurativen und keinen punitiven Zweck zu verfolgen.

- Die psychotherapeutische Massnahme darf das individualpräventive Potential der Strafe nicht verdrängen.

- Gegen die Missachtung ethischer Normen durch das therapeutische Personal ist ein disziplinarisches und strafrechtliches Regelwerk zu errichten. 